\title{
Distributed sensing with OFDR and its application to structural health monitoring
}

\author{
Hideaki Murayama ${ }^{1}$, Hirotaka Igawa ${ }^{2}$, Koji Omichi $^{3}$ and Yuichi Machijima ${ }^{4}$ \\ ${ }^{1}$ School of Engineering, The University of Tokyo, 7-3-1 Hongo, Bunkyo-ku, Tokyo, 113-8656 Japan \\ 2 Japan Aerospace Exploration Agency, 6-13-1 Osawa, Mitaka, Tokyo, 181-0015 Japan \\ ${ }^{3}$ Fujikura Ltd., 1440 Mutsuzaki, Sakura, Chiba, 285-8550 Japan \\ ${ }^{4}$ Lazoc Inc., 3-40-9 Hongo, Bunkyo-ku, Tokyo, 113-0033 Japan
}

\begin{abstract}
Optical fiber sensors are promised candidates as sensor elements in structural health monitoring (SHM). Especially fiber-optic distributed strain sensors that return a strain value as a function of linear position along an optical fiber have been attractive for people in the field of SHM. We have developed a distributed strain sensing system using long-length fiber Bragg gratings (FBGs), based on optical frequency domain reflectometry (OFDR). We employ long-length FBGs whose length is about $100 \mathrm{~mm}$ and the sensing region, in other words the gauge length, can be expanded up to more than $1 \mathrm{~m}$ by serially-cascaded long-length FBGs. This sensing system has the high spatial resolution of less than $1 \mathrm{~mm}$. In this paper the distributed sensing system with OFDR and its application to SHM are described.
\end{abstract}

Keywords: distributed sensing, long-length FBG, optical frequency domain reflectometry, structural health monitoring

\section{INTRODUCTION}

In structural health monitoring (SHM) the sensor elements are required to monitor a measurand which can be affected by damage or deterioration at positions interested. If the existence, location and type of damage in a structure are detected and identified based on measured results, we can assess the structural integrity which indicates how much the structure has strength margin. Then it can lead us to implement effective maintenance and safety operation. Effectiveness of the assessment of the structural integrity depends on the accuracy of the identification of damage. Frequently damage reconstructs a stress distribution around it or changes the stiffness of a structural member. This means that we can notice the existence of damage by detecting the fluctuation or the variation of the strain distribution. In some cases the location and type of damage can be also identified by analyzing the strain distribution. In a complicated structural system, such as aircraft, ship, or bridge, a lot of structural members are carrying loads as supporting each other to keep the whole structure. Therefore the ability of comprehensive monitoring in the structure is useful for SHM.

Optical fiber sensors are promised candidates as sensor elements in SHM. Especially fiber-optic distributed strain sensors that return a strain value as a function of linear position along an optical fiber have been attractive for people in the field of SHM because of the reason described above. Various distributed sensing techniques based on Brillouin scattering have been proposed for strain monitoring ${ }^{1-3}$. Murayama et al. applied Brillouin optical domain reflectometry (BOTDR) to strain monitoring of yacht hulls and at that time the spatial resolution and accuracy were $1 \mathrm{~m}$ and $\pm 30 \mu \varepsilon$, respectively ${ }^{4}$. Recently Song et al. achieved the spatial resolution of $1.6 \mathrm{~mm}$ using Brillouin optical correlation domain analysis (BOCDA) $)^{5}$. While fiber Bragg gratings (FBGs) with some interrogation systems are ordinarily employed for quasi-distributed strain or temperature measurements, they can be also applied to fully-distributed sensing along FBGs. A strain distribution along a chirped grating is measured by the phase-based sensing method or the low-coherence reflectometry ${ }^{6,7}$. We have developed a distributed strain sensing system using long-length FBGs, based on optical frequency domain reflectometry (OFDR) ${ }^{8}$. We confirmed by simulation that the spatial resolution of this system is less than $1 \mathrm{~mm}^{9}$. In this sensing system we employ long-length FBGs whose length is about $100 \mathrm{~mm}$ and the sensing region, in other words the gauge length, can be expanded up to $1,500 \mathrm{~mm}$ by serially-cascaded long-length $\mathrm{FBGs}^{10}$. These efforts to improve the spatial resolution of distributed sensing open recent development in the SHM field. 
In this paper the distributed sensing system with OFDR is described at first. Then as its application to SHM, strain monitoring and damage detection for an adhesively bonded joint is shown. Next, in SHM for a wing structure, the measurement results of the distributed strain monitoring in the composite wing model and load identification based on distributed strain data are shown.

\section{SENSING SYSTEM WIGH OFDR}

An arrangement of our sensing system is shown in Fig. 1. The distributed sensing system consists of a tunable laser source (TLS), two photo-optic detectors (PD1, PD2), three broad band reflectors (R1, R2, R3), three 3dB couplers (C1, C3, C3), three long-length FBGs (FBG1, FBG2, FBG3), an A/D converter (DAQ), and a computer. This arrangement is similar to the system with OFDR by Childers et al. ${ }^{11}$, except using the long-length FBGs. When a coherent source scans $\mathrm{R} 1$ and R2 as a function of wavelength or wavenumber, the resultant interference signal from PD1 is obtained and it is used as a trigger signal on DAQ to acquire the interference signal from PD2 at a constant wavenumber. The interference signal on PD2 is shown in Fig. 2. In this case the length of FBGs was about $100 \mathrm{~mm}$ and the interval was $10 \mathrm{~mm}$.

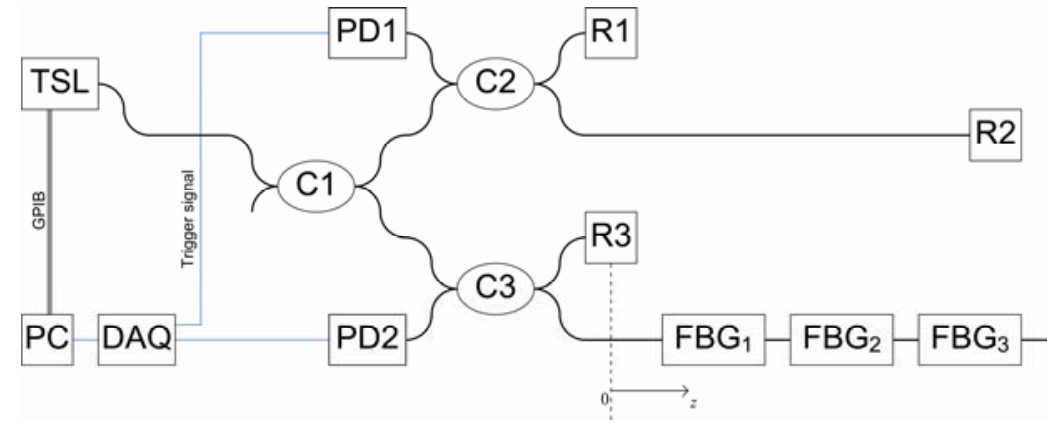

Figure 1. Sensing system.

In our sensing system we use a single processing technique based on short time Fourier transform (STFT) to map the strain profile along FBGs. In the case that STFT is applied to the interference signal shown in Fig. 2, time and frequency in usual STFT are corresponding to wavenumber and position, respectively. So by Fourier transform of the data within a window and sliding the window along the wavenumber axis, we can get a spectrogram as shown in Fig. 3 . The horizontal axis is converted from wavenumber to wavelength and the vertical axis represents the relative position, $z$, of Fig. 1 . The color depicts the intensity of the reflected light at wavelength, $\lambda$, and position, $z$. Wavelength spectrum of the reflected light at an arbitrary position can be extracted from the spectrogram. The spectrum at $4.7 \mathrm{~m}$ in Fig. 3 is shown in Fig. 4. The center wavelength can be determined by calculating the center of the full width at half maximum (FWHM) of the spectrum at each position. Figure 5 shows the center wavelength along FBGs. Finally strain distribution along FBGs is determined by the distribution of the center wavelength shift between the initial condition and the current one.

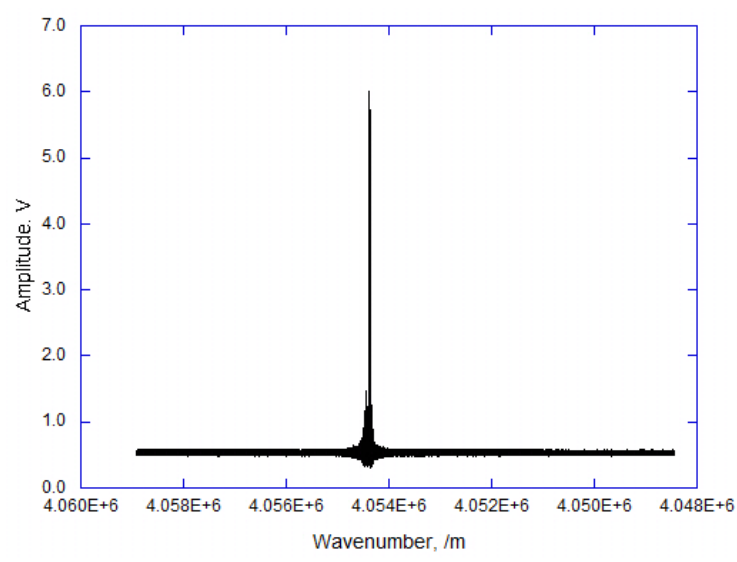

Figure 2. Measured interference signal on PD2.

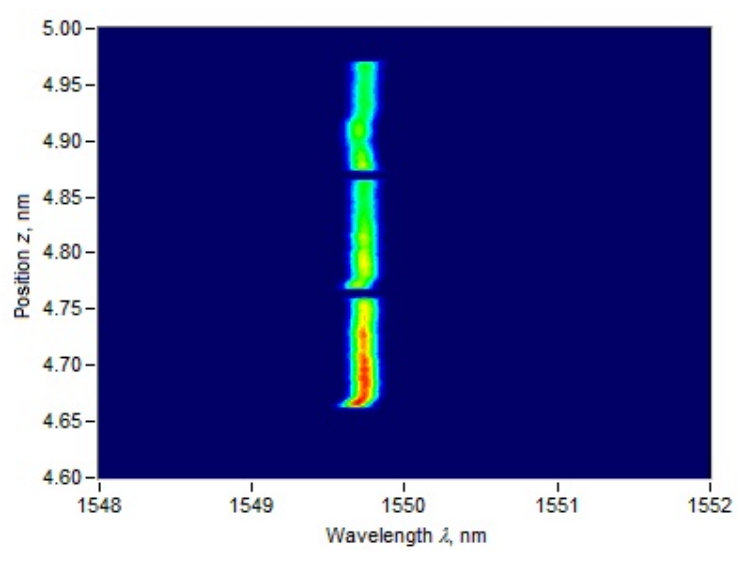

Figure 3. Spectrogram of measured interference signal. 


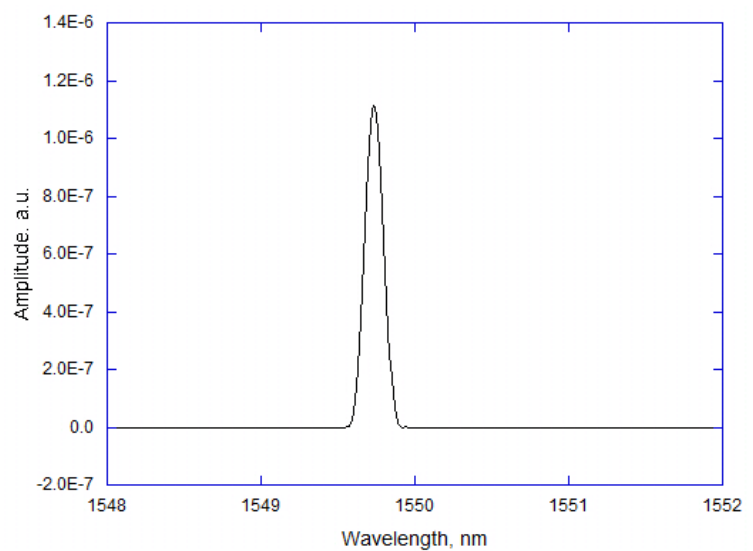

Figure 4. Spectrum at $4.7 \mathrm{~m}$ in spectrogram.



Figure 5. Center wavelength distribution in spectrogram.

\section{STRAIN MONITORING OF BONDED JOINT}

The use of adhesive bonding in composite structures is recently increasing in aerospace, marine, and automotive sectors because it has advantages over mechanically fastened joints, such as less source of stress concentrations, more uniform distribution of load and better fatigue properties ${ }^{12-14}$. We embedded a long-length FBG whose length is about 90 mm inside the adhesive of a single-lap joint and measure the strain distribution in the static tensile test. Dimensions of the test specimen and the location of the FBG sensor are shown in Fig. 6. The specimen is a single-lap joint of two aluminum palates (aluminum alloy 2017) adhered by epoxy adhesive (Araldite ${ }^{\circledR}$ 2011). The long-length FBG was set on the V-shaped grooved machined on the aluminum plate. The grating of the region A (40 mm) was bonded on the left adherend to measure the strain distribution of the left adherend which was outside the overlap region. The region B (30 $\mathrm{mm}$ ) was embedded inside the adhered section of the joint to measure strain distribution along the interface between the adherend and the adhesive. The region C (20 mm) was kept free to be used as reference part for compensation.

The specimen was subjected to static tensile loads and the strain distributions along the FBG were measured. We had seven load cases and the single-lap joint was broken at the load of 3,920 $\mathrm{N}$ as debonding in the case \#7. Measured strain distributions under the tensile loads in \#4 and \#7 are shown in Fig. 7 and 8, respectively. We confirmed the agreement between the measured strain in \#4 and the calculated one by finite element (FE) model without damage is excellent. Although it was too difficult to monitor the strain distribution inside the adhesive layer, we could successfully measure it. In the case \#7 distributed profile around the end of overlap differs from the case \#4. We also confirmed by using FE model with damage that debonding at the end of overlap caused the reconstruction strain distributions as shown in Fig. 8. It is important that we found the strain distribution around the end of overlap depends on the length of debonding, because it might be possible not only to know the existence of damage but also identify the location and type of damage.

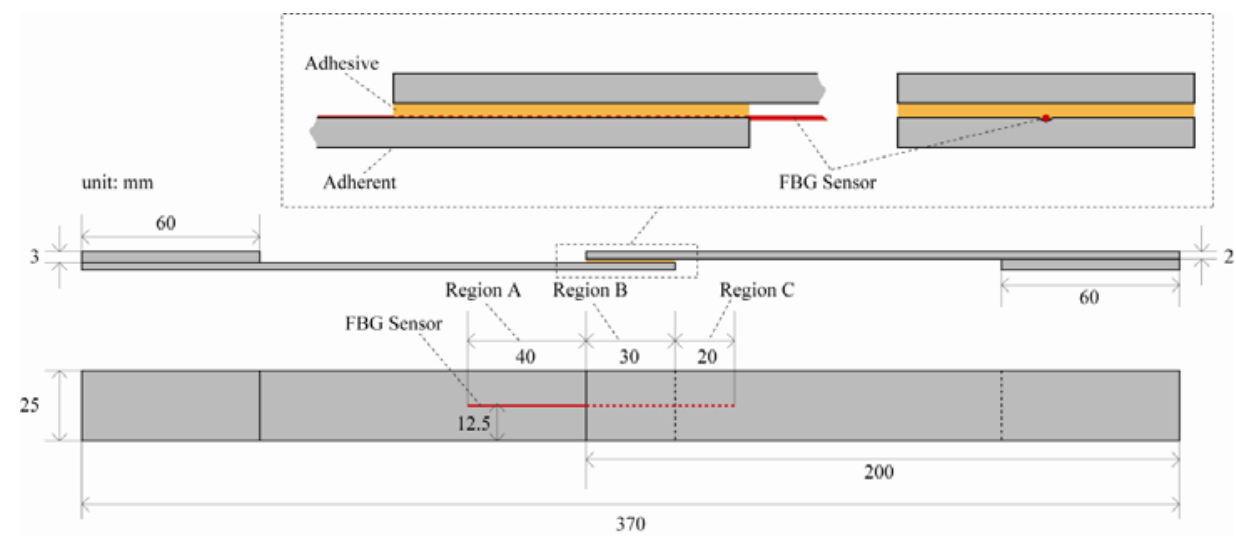

Figure 6. Dimensions of specimen and location of FBG sensor. 


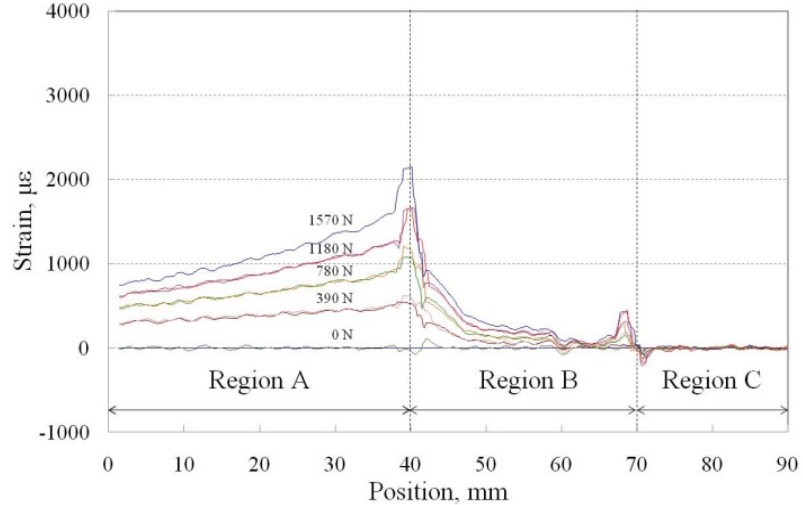

Figure 7. Strain distributions under the tensile loads (case \#4).

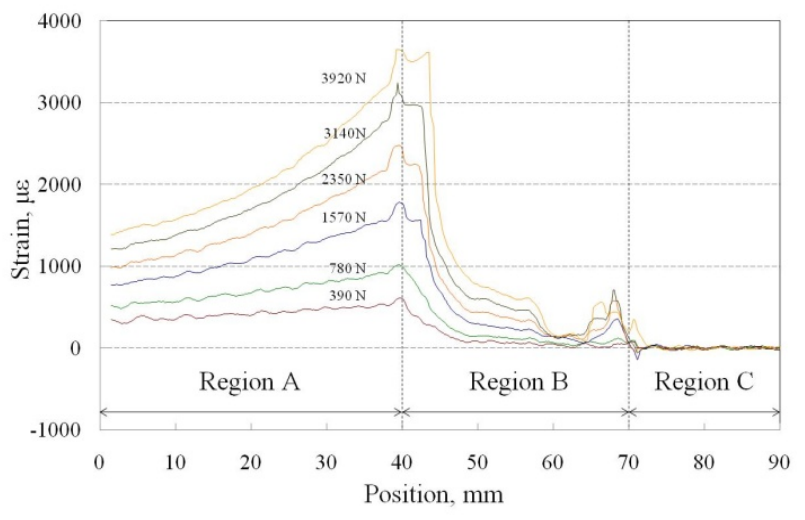

Figure 8. Strain distributions under the tensile loads (case \#7).

\section{SHM FOR WING STRUCTURE}

Wings of an aircraft are subjected to a distributed load to lift the body. The load varies depending on kinetic states, such as flight speed and direction, and environmental conditions, such as atmospheric pressure and its fluctuation, and so on. Distributed loads of the wings of an in-service aircraft can influence to in-flight safety and fatigue aging. Therefore if we know the loads of the in-service aircraft, we can increase the in-flight safety and the efficiency of the maintenance inspection for the aircraft which is designed based on damage tolerance. In general, however, the in-flight load distribution of a wing cannot be measure directly by sensors. It can be predicted by other structural conditions, such as strain and displacement, and this problem should be solved by an inverse analysis ${ }^{15,16}$. We have developed the prediction technique of the distributed loads on wing structures based on strain distributions measured by fiber-optic distributed sensors. In this technique, we are using finite element method (FEM) in order to enable integrated and organized operation of design and in-flight load prediction. In order to investigate the applicability of this technique to SHM, we implemented distributed strain monitoring of a composite wing structure by using the fiber-optic distributed sensing system $^{17}$ and carried out some simulations to confirm the accuracy of load identification by the inverse analysis ${ }^{18}$.

A composite wing box whose length is approximately $6 \mathrm{~m}$ was subjected to a series of load tests as shown in Fig. 9. Two types of FBGs were bonded to this structure as shown in Fig. 10. One was line-A, in which multiplexed FBGs with 10 $\mathrm{mm}$ length are arrayed in a $100 \mathrm{~mm}$ pitch. Seven lines of this type were bonded along the longitudinal direction of the wing in order to measure the overall deformation of the wing box. Each line had about 40 FBGs and the total number of FBGs was 257. The other was line-B, where a long-length FBG is installed. 14 lines of line-B were fixed adjacent to stress concentration zones where the stiffness is varying discontinuously. The gauge length of B1-B8 was about 300 mm and that of B9-B14 was about $500 \mathrm{~mm}$. The locations of these sensors were determined based on the stress distribution calculated by FEM. By using an optical channel selector which had 16 channels, we could monitor the strain distributions along each FBG line continuously.

In this paper, we show the measured results of Ch.1 and Ch.10. Ch.1 is connected to FBG line A-1, which was bonded straight along a stringer flange. Ch.10 is connected to FBG line B-3 and B-4, which were fixed along the edge of the rectangular area framed by ribs and stringers. Figure 11 shows the strain distributions measured by A-1. The horizontal axis represents the distance from R3 shown in Fig. 1. The whole length of the sensor array is approximately $4.1 \mathrm{~m}$. The figure shows that a larger strain occurred at the root of the wing. Moreover, the positions where a less strain occurred compared to nearby points almost coincided with the positions of ribs. This indicates that the change in the stiffness by structural reinforcement members was successfully measured. The measured strain showed an almost linear relation with the applied load. This result shows that the overall deformation of the wing box can be monitored by multiplexed FBG arrays using our sensing system.

Figure 12 shows the strain distributions measured by B-3 and B-4. Both 300 mm gauge length FBGs were bonded along a stringer at the front and the rear part, and bonded along a rib at the middle. We can see that tensile strain occurred at 
longitudinal directions of the wing whereas compression strain occurred at vertical directions. The amount became larger with the applied load and the ratio of the tensile and compression strain almost became equal to the Poisson's ratio. From these results, it can be concluded that the strain distribution of interest could be successfully measured with the high spatial resolution by long-length FBGs using our sensing system.
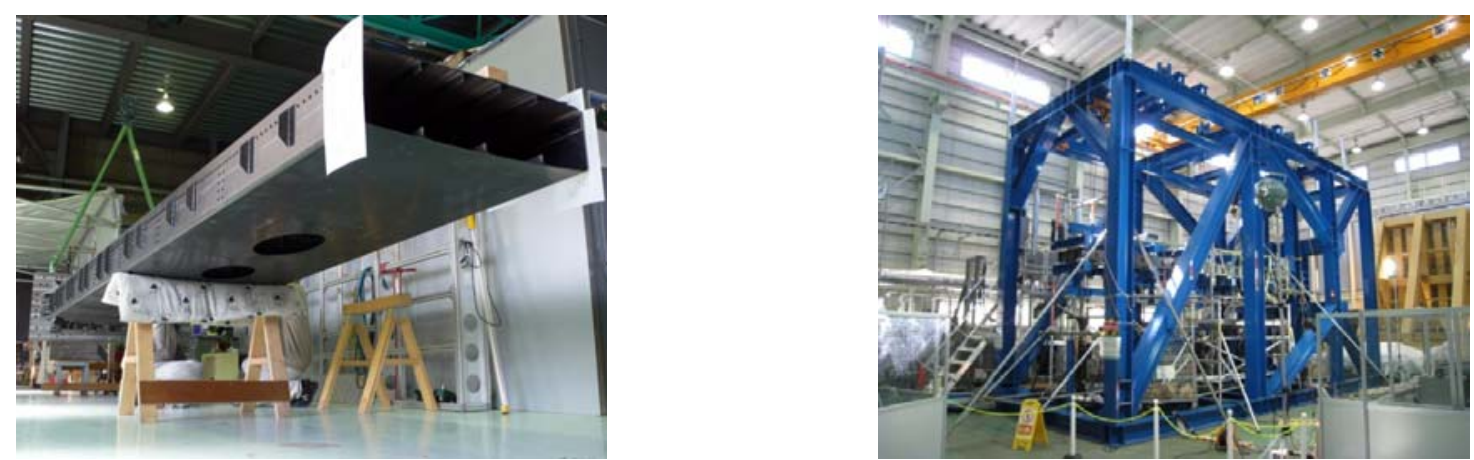

Figure 9. Composite wing box (left) and test equipment (right).

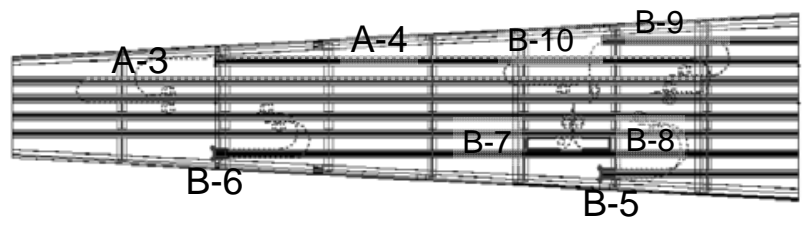

(a) upper side of inner skin



(c) lower side of inner skin

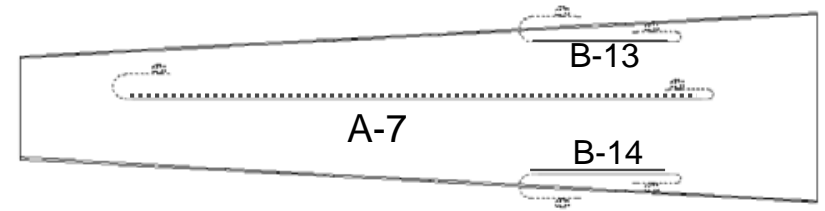

(b) upper side of outer skin

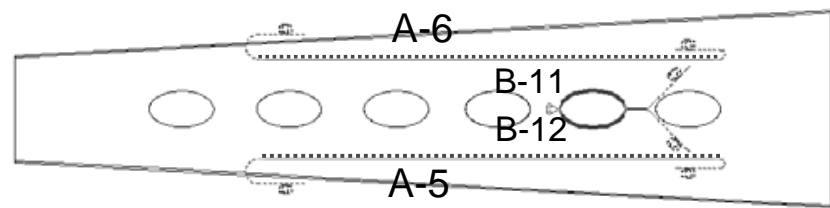

(d) lower side of outer skin

Figure 10. Sensor location in wing box.

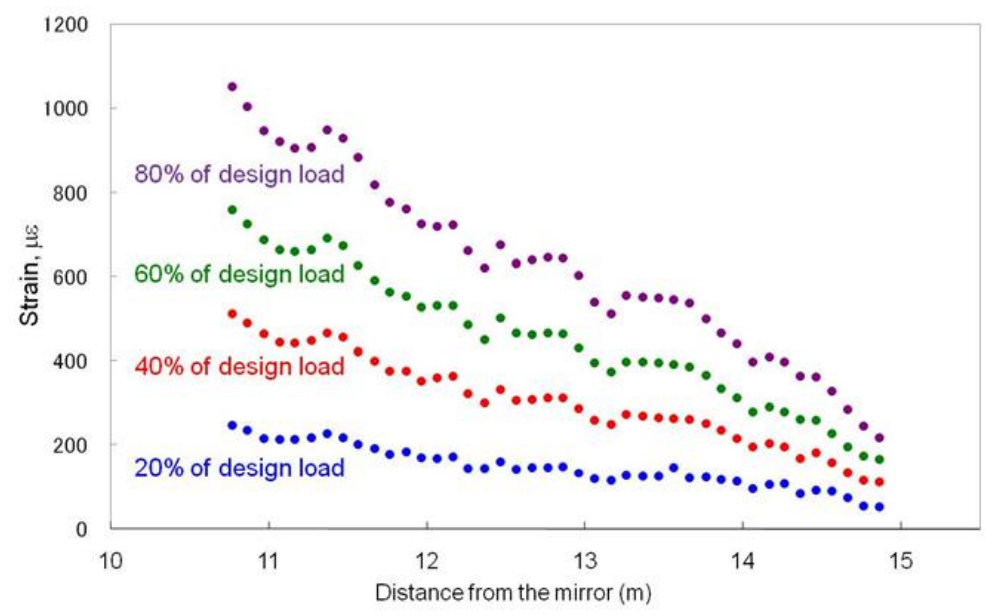

Figure 11. Strain distributions measured by A-1. 

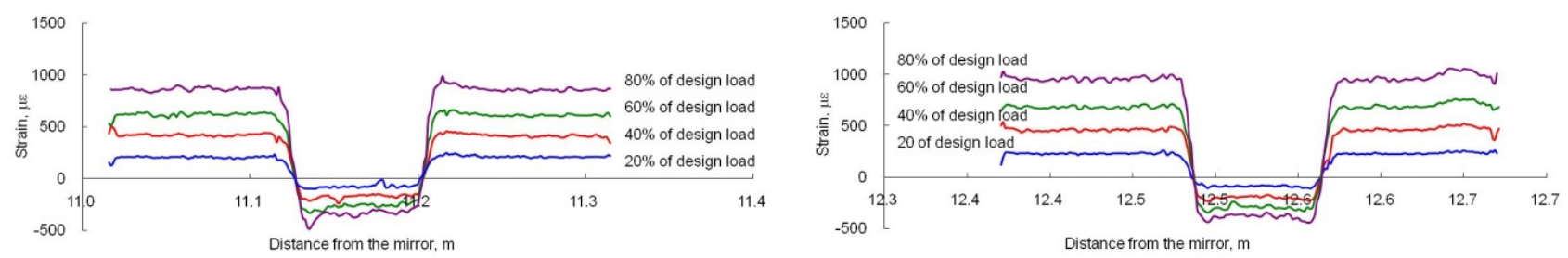

Figure 12. Strain distributions measured by B-1 (left) and B4 (right).

Finally we show the technique of load identification based on distributed strain data. Figure 13 shows a finite element model of the wing box shown in Fig. 9. We assumed that this structure is equipped with fiber-optic distributed strain sensors. The pressure distribution to be predicted and the pressure distribution predicted without error are shown in Fig. 14 and the agreement between them is excellent. This means that we can reconstruct strain and stress distributions in the whole wing box by applying the estimated load to the wing in FEM.

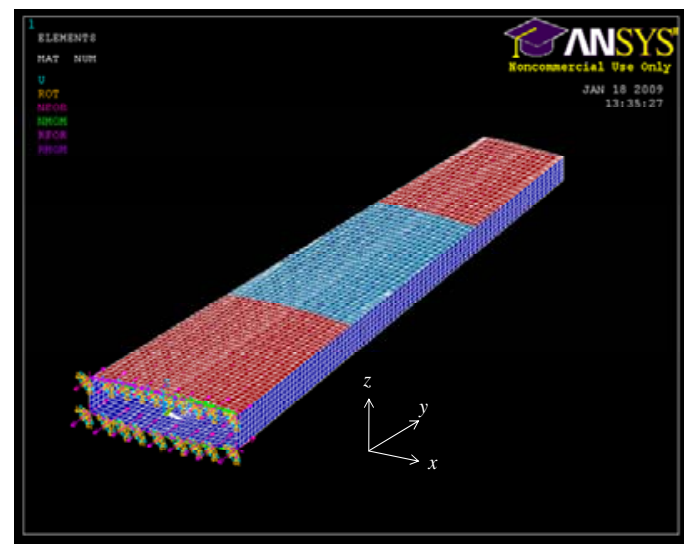

Figure 13. Finite element model of composite wing box.
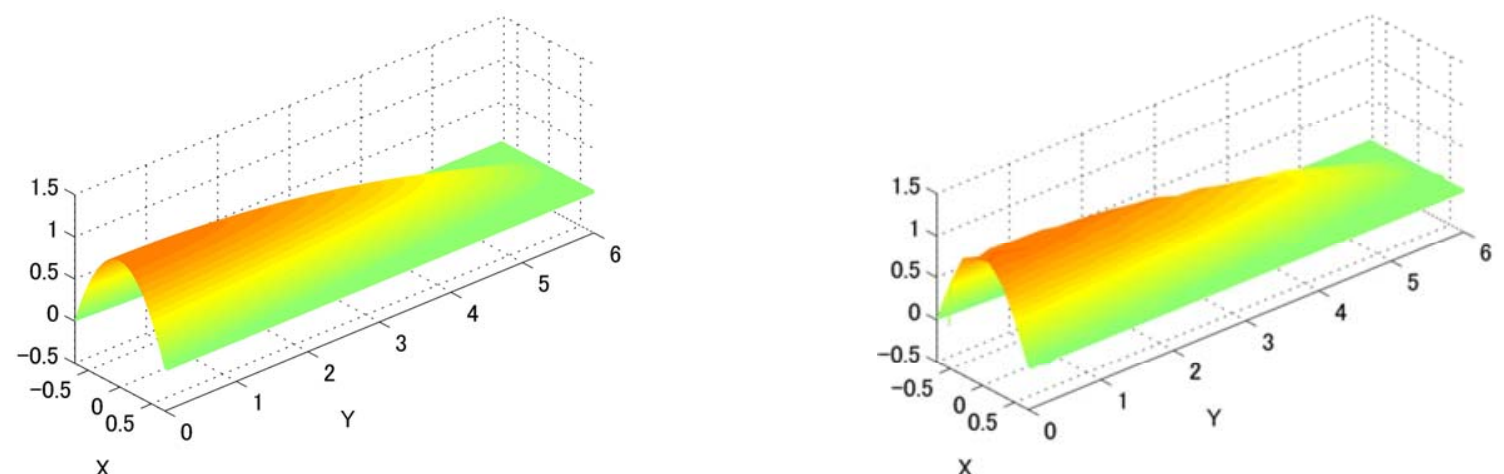

Figure 14. Pressure distributions to be predicted (left) and predicted (right).

\section{CONCLUSIONS}

In this paper we described the distributed sensing system with the high spatial resolution by using long-length FBGs and showed its application to SHM. The efforts to improve the spatial resolution and other functions of distributed sensing are opening recent development in the SHM field. In order to implement SHM for an in-service structure, we have to integrate the sensing system, the algorithm assessing the structural integrity, the installation method, and so on into an SHM system and prove the cost-benefit on maintenance works. This must be interdisciplinary work. 


\section{REFERENCES}

[1] Horiguchi, T., Kurashima, T. and Takeda, M., "Tensile strain dependence of Brillouin frequency shift in silica optical fiber,” IEEE Photonics Technol. Lett., 1(5), 107-108 (1989).

[2] Bao, X., Brown, A., DeMerchant, M. and Smith, J., "Characterization of the Brillouin-loss spectrum of single-mode fibers by use of very short (<10-ns) pulses," Opt. Lett., 24(8), 510-512 (1999).

[3] Hotate, K. and Hasegawa, T., "Measurement of Brillouin gain spectrum distribution along an optical fiber using a correlation-based technique -proposal, experiment and simulation," IEICE Trans. Electron., E83-C, 405-412 (2000).

[4] Murayama, H., Kageyama, K., Naruse, H., Shimada, A. and Uzawa, K., “Application of fiber-optic distributed sensors to health monitoring for full-scale composite structures,” J. Intell. Mater. Syst. Struct., 14(1), 3-13 (2003).

[5] Song, K.Y., He, Z., and Hotate, K., "Distributed strain measurement with millimeter-order spatial resolution based on Brillouin optical correlation domain analysis,” Opt. Lett., 31(17), 2526-2528 (2006).

[6] Volanthen, M., Geiger, H. and Dakin, J.P., “Distributed grating sensors using low-coherence reflectometry,” J. Lightwave Technol., 15(11), 2076-2082 (1997).

[7] Huang, S., Ohn, M.M. and Measure, R.M., "Phase-based Bragg intragrating distributed strain sensor,” Appl. Opt., 35(7), 1135-1142 (1996).

[8] Igawa, H., Murayama, H., Kasai, T., Yamaguchi, I., Kageyama, K. and Ohta, K., "Measurements of strain distribution with a long gauge FBG sensor using optical frequency domain reflectometry," Proc. SPIE, 5855, 547-550 (2005).

[9] Igawa, H., Ohta, K., Kasai, T., Yamaguchi, I., Murayama, H., and Kageyama, K., "Distributed measurements with a long gauge FBG sensor using optical frequency domain reflectometry (1st report, system investigation using optical simulation model),” JSME Int J., Ser. A, 2(9), 1242-1252 (2008).

[10] Igawa, H., Murayama, H., Nakamura, T., Yamaguchi, I., Kageyama, K., Uzawa, K., Wada, D., Ohsawa, I., Kanai, M. and Omichi, K., "Measurement of distributed strain and load identification using $1500 \mathrm{~mm}$ gauge length FBG and optical frequency domain reflectometry,” Proc. SPIE, 7503, 750351-1-750351-4 (2009).

[11] Childers, B.A., Froggatt, M.E., Allison, S.G., Moore, T.C., Hare, D.A., Batten, C.F. and Jegley, D.C., "Use of 3000 Bragg grating strain sensors distributed on four eight-meter optical fibers during static load tests of a composite structure," Proc. SPIE, 4332, 133-142 (2001).

[12] Kwakernaak, A. and Hofstede, J.C.J., "Adhesive bonding: providing improved fatigue resistance and damage tolerance at lower cost,” SAMPE Journal, 44(5), 6-15 (2008).

[13] Cao, J. and Grenstedt, L., "Design and testing of joints for composite sandwich/steel hybrid ship hull," Composites Part A, 35(9), 1091-1105 (2004).

[14] Fessel, G., Broughton, J.G., Fellows, N.A., Durodola, J.F. and Hutchinson, A.R., "Evaluation of different lap-shear joint geometries for automotive applications,” Int. J. Adhes. Adhes., 27(7), 574-583 (2007).

[15] Coates, C.W., Thamburai, P. and Kim, C.J., "An inverse method for selection of Fourier coefficients for flight load identification,” AIAA 2005-2183 (2005).

[16] Shkarayev, S., Krashanitsa, R. and Tessler, A., "An inverse interpolation method utilizing in-flight strain measurements for determining loads and structural response of aerospace vehicles," Proc. of 3rd IWSHM, 336-343 (2001).

[17] Murayama, H., Igawa, H., Yamaguchi, I., Hirano, Y., Aoki, Y., Kageyama, K., Uzawa, K., Akiyama, G., Ohara, K., Nunotani, K., Azemoto, M. and Omichi, K., "Distributed strain monitoring of composite wing structure by FBG sensors,” 13th US-Japan Conference on Composite Materials, CD-ROM (2008).

[18] Murayama, H., Akiyama, G., Igawa, H., Nakamura, T., Kageyama, K., Uzawa, K., Hirano, Y. and Aoki, Y., "Application of inverse analysis of distributed load with strain sensors to wing structures," Structural Health Monitoring 2009, DEStech Publications, Inc., 75-82 (2009). 